\title{
New infrared star clusters and candidates in the Galaxy detected with 2MASS
}

\author{
C. M. Dutra ${ }^{1,2}$ and E. Bica ${ }^{1}$ \\ 1 Universidade Federal do Rio Grande do Sul, IF, CP 15051, Porto Alegre 91501-970, RS, Brazil \\ 2 Instituto Astronomico e Geofisico da USP, CP 3386, São Paulo 01060-970, SP, Brazil
}

Received 17 April 2001 / Accepted 5 July 2001

\begin{abstract}
A sample of 42 new infrared star clusters, stellar groups and candidates was found throughout the Galaxy in the 2MASS $J, H$ and especially $K_{\mathrm{S}}$ atlases. In the Cygnus X region 19 new clusters, stellar groups and candidates were found as compared to 6 such objects in the previous literature. Colour-Magnitude Diagrams using the 2MASS Point Source Catalogue provided preliminary distance estimates in the range $1.0<d_{\odot}<1.8 \mathrm{kpc}$ for 7 Cygnus X clusters. Towards the central parts of the Galaxy 7 new IR clusters and candidates were found as compared to 61 previous objects. A search for prominent dark nebulae in $K_{\mathrm{S}}$ was also carried out in the central bulge area. We report 5 dark nebulae, 2 of them are candidates for molecular clouds able to generate massive star clusters near the Nucleus, such as the Arches and Quintuplet clusters.
\end{abstract}

Key words. Galaxy: open clusters and associations: individual

\section{Introduction}

The digital Two Micron All Sky Survey (2MASS) infrared atlas (Skrutskie et al. 1997 - web interface http://www.ipac.caltech.edu/2mass/) can provide a wealth of new objects for future studies with large telescopes, a role similar to that played in the optical by the Palomar and ESO/SERC Schmidt plate Atlases in past decades. Regarding Galactic IR star clusters two basic classes can be identified in the 2MASS survey: (i) resolved embedded clusters in nearby complexes such as those in Perseus, Orion and MonR2 which were studied by means of star counts (Carpenter 2000), and (ii) partly resolved clusters or candidates located in more distant parts of the Galaxy, such as towards the Centre (Dutra \& Bica 2000a, hereafter Paper I).

As the IR search for new objects progresses, it is worth recalling how the development of overall optical catalogues of similar objects occurred. For comparison purposes, no survey or search of optical star clusters and nebulae has ever been complete. The samples currently available in overall optical catalogues arise from many contributions, and in the following we mention some examples. The open cluster catalogues as compiled by Alter et al. (1970) and Lyngå (1987) had major contributions from short communications including small lists of newly found objects. Despite a systematic search for faint

Send offprint requests to: C. M. Dutra,

e-mail: dutra@iagusp.usp.br clusters on the Palomar plates like that generating the Berkeley clusters (Setteducati \& Weaver 1962), or the individual lists which generated the ESO catalogue (Lauberts 1982 and references therein), until quite recently new star clusters and candidates both on the Palomar and ESO/SERC Schmidt plates could still be found (e.g. the 6 new objects in Saurer et al. 1994). All catalogues of open stellar clusters are biased in one way or the other, and their statistical value is uncertain. Concerning optical nebulae, the northern/equatorial Sh2 catalogue (Sharpless 1959) had important complementary contributions, e.g. the BFS objects (Blitz et al. 1982). In the Magellanic Clouds the same process has taken place over decades (e.g. Bica \& Dutra 2000; Bica et al. 1999 and references therein).

In the present study we make use of the 2MASS survey in the $J(1.25 \mu \mathrm{m}), H(1.65 \mu \mathrm{m})$ and $K_{\mathrm{s}}(2.17 \mu \mathrm{m})$ bands to search for new IR star clusters in the Galaxy, in particular towards the Cygnus X region. In Sect. 2 we discuss the search criteria and present the results. In Sect. 3 we discuss the angular distribution of the sample and preliminary implications of the cluster detections, as well as of some newly found dark and bright nebulae. Finally, the concluding remarks are given in Sect. 4.

\section{Search for IR star clusters and candidates in the 2MASS Atlas}

The search was carried out in selected 2MASS areas with evidence of star formation, while that of Paper I was 
systematic in a specific area. The selection follows procedures for cross-identifications and new detections of extended objects as outlined in Bica \& Schmidt (1995) and Bica et al. (1999).

The $J H K_{\mathrm{s}}$ images were obtained by means of the 2MASS Survey Visualization \& Image Server facility in the web interface http://irsa.ipac.caltech.edu/. The $K_{\mathrm{s}}$ band allows one to probe deeper in more absorbed regions, and the $J$ and $H$ bands were used mostly as control images for the presence of bright stars, and as comparisons to estimate how reddened the objects could be, and what to expect in optical bands. We also checked for possible optical counterparts using 1st (DSS) and 2nd (XDSS) generation digitized sky survey extractions. In the generated sky charts we were also guided by the presence of IRAS sources in general (Beichman et al. 1988) and/or ultracompact H II regions (Kurtz et al. 1994). We also checked the objects for possible relationship to dark nebulae (Lynds 1962). For some objects the absorption is patchy even in the $K_{\mathrm{s}}$ band, so that one cannot rule out the possibility of an enhancement of field stars - a small dust window.

The present work was not exhaustive and it is not intended to be complete in any sense in the currently available 2MASS second incremental release Atlas areas. Nevertheless, some preliminary detection rates can be inferred in view of future searches. We focused our attention mainly on the Cygnus X area, but we also continued the exploration of central directions in the Galaxy, as well as some other samplings along the disk.

\subsection{Targets in Cygnus $X$}

In the Cygnus $\mathrm{X}$ area we concentrated efforts on the large angular size continuum sources (Downes \& Rinehart 1966; Wendker 1970), thus avoiding many small discrete sources related to the thermal emission fine structure (Wendker et al. 1991). Indeed, optical H II regions often show embedded clusters which are small with respect to the overall emission extent and detailed structure of the complex. Out of the 21 DR sources 11 are now known to harbour a star cluster or stellar aggregate (Sect. 3.1). Wendker's (1970) continuum sources basically include those in Downes \& Rinehart (1966), and several of the additional clusters and aggregates in the area (Table 1, Sect. 3.1) are related to them. Excluding those related to DR sources, the cluster and aggregate detection rate is $\approx 1: 4$ for the remaining Wendker (1970) sources, which in turn suggests that most of the remaining radio continuum sources are related to the details of the continuum distribution, like thermal shells from individual massive stars, ridges and some nonthermal sources. Alternatively, some non-detections might be due to high $K_{\mathrm{s}}$ absorptions for deeply embedded clusters in dense molecular cores as seen in isolated relatively high Galactic latitute complexes (Dutra \& Bica 2000b and references therein).

\subsection{Targets in central parts of the Galaxy}

Towards the central parts of the Galaxy we based our search mostly on the radio continuum catalogue by Kuchar \& Clark (1997), and the radio recombination line catalogues by Caswell \& Haynes (1987) and Lockman (1989). For such directions a preliminary estimate suggests that stellar enhancements in the $K_{\mathrm{s}}$ band are detectable for 1:5 radio HII regions. This might be explained by important intervening dust contributions for such directions (Dutra \& Bica 2000b), combined to the internal absorption arising from the cluster dust complex itself. Star crowding is an additional difficulty as compared to the Cygnus X region.

\subsection{Targets along the disk}

Towards the anticentre and southern Milky Way the search was mostly aimed at a few specific entries from optical nebula catalogues (Sharpless et al. 1959; Rodgers et al. 1960; Blitz et al. 1982). Some of these H II regions or complexes have known optical open clusters, e.g. $\operatorname{Tr} 14, \operatorname{Tr} 16$ and their neighbours in the $\eta$ Carinae Complex (Feinstein 1982; Vázquez et al. 1996 and references therein), or the IR massive central star cluster in the RCW 38 Complex as recently shown in detail by means of VLT/ISAAC imaging (Moorwood et al. 1998). In the present study one additional cluster was detected in each of these optical complexes (Sect. 3.3). Perhaps the best example of detection possibilities in optical H II regions is the Sh2-254/258 Complex. Five prominent discrete H II regions led to 4 IR clusters, only one was previously reported (Sect. 3.3).

\subsection{Results}

A total of 42 newly detected IR star clusters, stellar groups and candidates are given in Table 1. By columns: (1) a running number identification, (2) and (3) Galactic coordinates, (4) and (5) J2000.0 equatorial positions, (6) and (7) major and minor angular dimensions, and (8) remarks such as degree of resolution, classification, duplicity, and related nebulae and other sources. We distinguish star clusters from less populous and/or less dense possible physical systems, which are referred to as stellar groups. Angular diameters of the objects in Table 1 are typically $1^{\prime}-2^{\prime}$, thus suitable for deep images in large telescopes.

We illustrate in Fig. 1 the object 13 from Table 1, which is the IR resolved star cluster in the HII region DR 22 in Cygnus X. This cluster is surrounded by a related sparse group of stars (object 14). Recently an IR stellar group or aggregate was discussed in the $\mathrm{HII} /$ photodissociation region DR 18 in Cygnus $\mathrm{X}$ (Comerón \& Torra 1999). A newly found IR nebula $\left(\alpha(2000)=20^{\mathrm{h}} 39^{\mathrm{m}} 25.9^{\mathrm{s}}, \delta(2000)=41^{\circ} 20^{\prime} 02^{\prime \prime}\right)$ is also present in Fig. 1. In this nebula no candidate cluster was detected, since in the 2MASS J image a single bright star is centred in the nebula. A similar IR nebula was studied by Comerón \& Torra (1999) in DR 18. The candidate clusters 


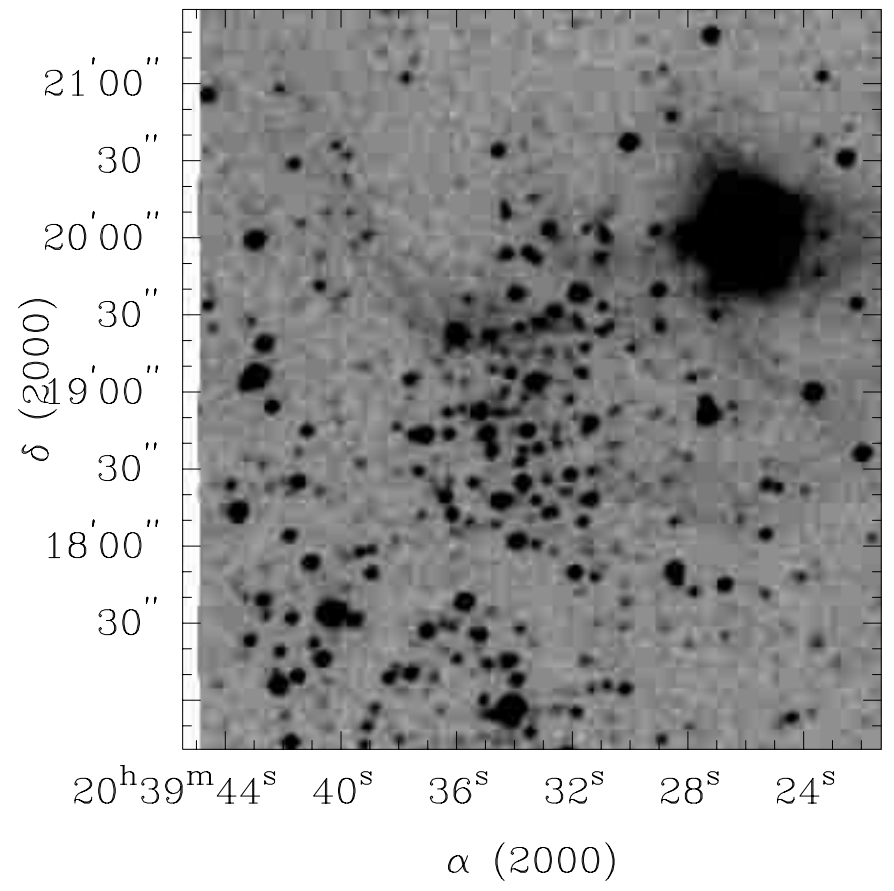

Fig. 1. $5^{\prime} \times 5^{\prime}$ MASS $K_{\mathrm{s}}$ image of the IR cluster $(\alpha=$ $20^{\mathrm{h}} 39^{\mathrm{m}} 34^{\mathrm{s}}$ and $\left.\delta=41^{\circ} 19^{\prime} 05^{\prime \prime} \mathrm{J} 2000\right)$ in the radio HII region DR 22 . A newly identified IR compact nebula is located at $\approx 2^{\prime}$ to the northwest.



Fig. 2. $3^{\prime} \times 3^{\prime}$ MASS $K_{\mathrm{s}}$ image of the predominantly unresolved cluster candidate $\left(\alpha=17^{\mathrm{h}} 30^{\mathrm{m}} 28^{\mathrm{s}}\right.$ and $\delta=-34^{\circ} 41^{\prime} 30^{\prime \prime}$ $\mathrm{J} 2000$ ) in the radio HII region G353.4-0.4.

in Table 1 are predominantly unresolved objects and/or much embedded in nebulae in the IR bands. Figure 2 illustrates the case of Object 40 , which is related to the radio H II region G353.4-0.4.

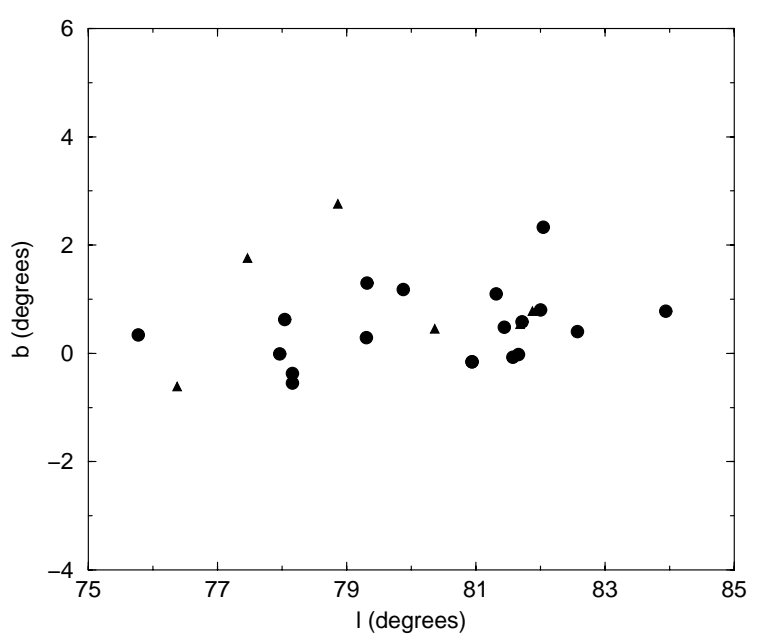

Fig. 3. Cygnus X area: distribution of new IR clusters, stellar groups or candidates (circles) compared to those already known (triangles).

\section{Discussion}

\subsection{Cygnus $X$}

The Galactic longitude distribution in Table 1 indicates many new objects around $l \approx 80^{\circ}$ which corresponds to the Local Arm plunge into Cygnus. We have extensively surveyed this region with 2MASS which corresponds to the HII region/molecular complexes collectively known as Cygnus X (Downes \& Rinehart 1966). The direction of Cygnus $\mathrm{X}$ is very complex. By increasing the angular resolution the number of discrete radio continuum sources increased from about 20 in Downes \& Rinehart's study to nearly 800 in Wendker (1984). Recombination lines (e.g. Piepenbrink \& Wendker 1988) show that most sources are related to the Local Arm, but owing to the tangent orientation, kinematical distances basically cannot be inferred for $d_{\odot}<3 \mathrm{kpc}$. The association Cyg OB2 is projected near the centre of Cygnus $\mathrm{X}$ and a fundamental question is whether a coherent local physical phenomenon relates all these structures or whether they are simply line-of-sight projections (Wendker et al. 1991; Odenwald \& Schwartz 1993, and references therein). Cyg OB2 itself is an unusually rich and compact association (Knödlseder 2000). The depth structure of Cygnus $\mathrm{X}$ is not yet known in detail, but available estimates as reviewed by Odenwald \& Schwartz (1993) point to significant effects $\left(0.7<d_{\odot}<2.5 \mathrm{kpc}\right.$ or more).

The angular distribution of Table 1 objects in the Cygnus $\mathrm{X}$ area (Fig. 3) is more elongated in longitude than in latitude (3:1). Assuming an average distance of $1.6 \mathrm{kpc}$, the objects are distributed along a region of $\approx 250 \times 85 \mathrm{pc}$. This size is comparable to those of the largest OB associations, but the total number of 25 IR star clusters and stellar groups is remarkable. The new objects are 19, while previously known in the area were 6 : (i) W $75 \mathrm{~S}$ (or DR 21) IR cluster (Hodapp 1994), (ii) W 75N IR cluster (Moore et al. 1988, Hodapp 1994); (iii) Sh2-106 IR cluster (Hodapp \& Rayner 1991), (iv) IRAS 20188+3928 
Table 1. New infrared star clusters, stellar groups or candidates.

\begin{tabular}{|c|c|c|c|c|c|c|c|}
\hline Object $^{* *}$ & $\begin{array}{r}\ell \\
\left({ }^{\circ}\right) \\
\end{array}$ & $\begin{array}{r}b \\
\left({ }^{\circ}\right) \\
\end{array}$ & $\begin{array}{r}\alpha(2000) \\
\text { hh:mm:ss.s } \\
\end{array}$ & $\begin{array}{c}\delta(2000) \\
\circ::^{\prime \prime}\end{array}$ & $D_{\text {, }}$ & ${ }_{1} d$ & Remarks* \\
\hline 1 & 2.20 & 3.45 & $17: 37: 40.7$ & $-25: 15: 03$ & $\overline{1.9}$ & 1.7 & resolved cluster or dust window, related to LDN74? partly optical \\
\hline 2 & 3.73 & 3.96 & $17: 39: 20.1$ & $-23: 41: 00$ & 1 & 0.6 & resolved stellar group, related to LDN114? partly optical \\
\hline 3 & 66.96 & -1.28 & 20:04:52.9 & 29:11:45 & 2 & 2 & mostly a resolved cluster in nebula IC $4955=\mathrm{BFS} 2 \mathrm{c}$, in complex IC 4954 \\
\hline 4 & 67.00 & -1.22 & 20:04:45.4 & 29:15:05 & 0.5 & 0.4 & partly resolved stellar group in nebula BFS2a, in complex IC4954 \\
\hline 5 & 75.77 & 0.34 & $20: 21: 41.8$ & $37: 25: 50$ & 1.7 & 1.7 & $\begin{array}{l}\text { cluster and/or IR nebula in radio HII region } \mathrm{G} 75.8+0.4 \text {,rel. to } \mathrm{OH} \text { maser ON2, } \\
\text { backgr. of cluster Be87 }\end{array}$ \\
\hline 6 & 77.96 & -0.01 & $20: 29: 36.9$ & 39:01:15 & 3 & 3 & partly resolved cluster in radio HII region DR9 \\
\hline 7 & 78.04 & 0.62 & $20: 27: 13.0$ & $39: 26: 56$ & 3.5 & 3 & resolved cluster in radio HII region DR6 \\
\hline 8 & 78.16 & -0.37 & $20: 31: 45.4$ & $38: 58: 00$ & 2 & 2 & partly resolved cluster in Radio HII region DR13 \\
\hline 9 & 78.16 & -0.55 & $20: 32: 27.8$ & $38: 51: 26$ & 1.4 & 1.2 & partly resolved cluster related to IRAS $20306+3841$, in radio complex W69 \\
\hline 10 & 79.30 & 0.29 & $20: 32: 29.0$ & $40: 16: 30$ & 2.5 & 2.0 & $\begin{array}{l}\text { mostly a resolved cluster in radio HII region G79.306+0.282 in DR } 15 \text {, related to } \\
\text { IRAS20306+4005 }\end{array}$ \\
\hline 11 & 79.31 & 1.30 & 20:28:09.7 & $40: 52: 42$ & 3.5 & 3.0 & mostly a resolved cluster in Radio HII region DR7, related to IRAS $20264+4042$ \\
\hline 12 & 79.87 & 1.18 & $20: 30: 28.0$ & $41: 15: 48$ & 1.8 & 1.8 & resolved stellar group in radio HII region DR 11 , related to IRAS $20286+4105$ \\
\hline 13 & 80.94 & -0.16 & 20:39:34.0 & 41:19:05 & 2.3 & 1.7 & resolved cluster in radio HII region DR22, in stellar group 14 \\
\hline 14 & 80.94 & -0.16 & 20:39:33.0 & $41: 18: 46$ & 4 & 3 & $\begin{array}{l}\text { resolved stellar group in radio HII region DR22, includes cluster } 13 \text { and a compact } \\
\text { nebula }\end{array}$ \\
\hline 15 & 81.31 & 1.10 & $20: 35: 23.0$ & $42: 22: 05$ & 2.9 & 2.2 & resolved cluster in radio HII region DR17 \\
\hline 16 & 81.44 & 0.48 & $20: 38: 29.0$ & $42: 06: 25$ & 2.3 & 2.0 & $\begin{array}{l}\text { mostly a resolved cluster, in radio complex W75, related to radio HII region } \\
\text { G81.5+0.6? }\end{array}$ \\
\hline 17 & 81.57 & -0.07 & $20: 41: 15.9$ & 41:51:51 & 1.7 & 1.7 & resolved cluster in radio HII region DR23 \\
\hline 18 & 81.66 & -0.02 & $20: 41: 20.1$ & $41: 58: 22$ & 1.8 & 1.3 & resolved stellar group at the edge of radio HII region DR23, related to DR23? \\
\hline 19 & 81.71 & 0.58 & $20: 38: 56.8$ & $42: 22: 45$ & 2 & 2 & partly resolved stellar group in radio HII region W75, pair with W75S IR cluster \\
\hline 20 & 82.00 & 0.80 & $20: 38: 37.0$ & $42: 39: 24$ & 1.5 & 1.5 & mostly a resolved cluster in radio HII region W75N, pair with W75N IR cluster \\
\hline 21 & 82.04 & 2.33 & $20: 32: 21.8$ & 43:41:05 & 2.5 & 2.0 & resolved cluster in radio HII region G82.0+2.3, related to radio HII region DR $16 ?$ \\
\hline 22 & 82.57 & 0.40 & $20: 42: 33.5$ & $42: 56: 50$ & 1.7 & 1.5 & partly resolved cluster in radio HII region G82.6+0.4 \\
\hline 23 & 83.94 & 0.78 & $20: 45: 37.6$ & 44:15:21 & 1.8 & 1.8 & partly resolved stellar group in radio HII region G83.941+0.781 \\
\hline 24 & 173.48 & 2.45 & $5: 39: 12.5$ & $35: 45: 53$ & 1.2 & 1.2 & partly resolved cluster in HII region Sh2-233SE, pair with Sh2-233SE IR Cluster \\
\hline 25 & 182.05 & 0.42 & 5:52:04.9 & $27: 23: 32$ & 3.5 & 3.5 & resolved cluster or dust window, related to IRAS $05489+2723$ ?, partly optical \\
\hline 26 & 186.13 & 2.59 & $6: 09: 28.2$ & $24: 55: 40$ & 4 & 3 & resolved stellar group related to IRAS $06063+2456$, partly optical \\
\hline 27 & 189.23 & 0.90 & 6:09:31.0 & $21: 23: 57$ & 2 & 2 & resolved stellar group related to IRAS $06065+2124$ \\
\hline 28 & 189.69 & 0.72 & 6:09:46.9 & $20: 54: 44$ & 2.5 & 2.5 & resolved stellar group related to IRAS $06067+2055$ \\
\hline 29 & 191.92 & 0.82 & $6: 14: 45.1$ & 19:00:28 & 2.5 & 2 & resolved cluster in nebula BFS52, related to IRAS $06117+1901$ \\
\hline 30 & 192.54 & -0.15 & $6: 12: 24.9$ & $17: 59: 28$ & 0.9 & 0.9 & partly resolved cluster in HII region Sh2-254 \\
\hline 31 & 192.60 & -0.12 & $6: 12: 36.6$ & $17: 56: 55$ & 1.7 & 1.7 & mostly a resolved cluster in HII region Sh2-256 \\
\hline 32 & 192.72 & 0.03 & $6: 13: 28.0$ & $17: 55: 27$ & 2 & 2 & partly resolved cluster in HII region Sh2-258 \\
\hline 33 & 194.93 & -1.20 & $6: 13: 21.2$ & $15: 23: 56$ & 1 & 1 & mostly an unresolved cluster related to IRAS $06104+1524 \mathrm{~A}$, pair with stellar group 34 \\
\hline 34 & 194.94 & -1.22 & $6: 13: 16.0$ & $15: 22: 30$ & 1.3 & 1 & mostly a resolved stellar group related to IRAS $06103+1523$, pair with cluster 33 \\
\hline 35 & 218.74 & 1.85 & $7: 08: 39.0$ & $-4: 19: 07$ & 0.6 & 0.4 & mostly unresolved cluster in HII region IC $466=$ Sh2-288 \\
\hline 36 & 267.72 & -1.09 & 8:58:05.0 & $-47: 22: 40$ & 1.2 & 1.2 & partly resolved cluster in reflection nebula vdBH-RN26, in RCW38? \\
\hline 37 & 287.81 & -0.82 & 10:45:54.0 & $-59: 56: 58$ & 1.5 & 1.5 & partly resolved cluster in reflection nebula vdBH-RN43, in $\eta$ Carinae Complex? \\
\hline 38 & 351.61 & 0.17 & $17: 23: 23.4$ & $-35: 53: 44$ & 0.4 & 0.3 & mostly unresolved cluster in radio HII region G351.6+0.2 \\
\hline 39 & 352.86 & -0.20 & $17: 28: 19.1$ & $-35: 04: 15$ & 0.3 & 0.3 & mostly unresolved cluster candidate in G352.866-0.199 \\
\hline 40 & 353.42 & -0.36 & $17: 30: 28.2$ & $-34: 41: 30$ & 1.7 & 1.5 & mostly unresolved cluster in radio HII region G353.4-0.4 \\
\hline 41 & 354.67 & 0.48 & $17: 30: 24.0$ & $-33: 11: 15$ & 1 & 0.7 & partly resolved cluster in radio HII region G354.664+0.470 \\
\hline 42 & 359.28 & -0.25 & $17: 44: 52.7$ & $-29: 40: 48$ & 2 & 1.7 & resolved cluster in radio HII region G359.3-0.3, dust window? \\
\hline
\end{tabular}

Notes: * Designations and related object types are-BFS: HII regions and/or reflection nebulae (Blitz et al. 1982); Sh2: HII regions (Sharpless 1959); RCW: HII regions (Rodgers et al. 1960); DR: radio HII regions in Cygnus X (Downes \& Rinehart 1966); G: radio HII regions (see text); vdBH-RN: reflection nebulae (van den Bergh \& Herbst 1975); LDN: dark nebulae (Lynds 1962); ON: OH maser (Norris et al. 1980, and references therein); IRAS sources (Beichman et al. 1988).

** After this manuscript had been completed we learned about a paper on Cygnus X (Comerón \& Torra 2001) that covers partially the same objects.

IR cluster (Hodapp 1994), (v) BD $+40^{\circ} 4124$ stellar aggregate (Hillenbrand et al. 1995), (vi) DR 18 stellar aggregate (Comerón \& Torra 1999).

Embedded clusters can occur in pairs or triplets (e.g. NGC 1333NE and NGC 1333SW IR clusters - Lada et al. 1996, and the subclusterings A, B and C of IRAS 20050+2720 - Chen et al. 1997). Combining new and previous IR clusters and stellar groups in Cygnus $\mathrm{X}$ there occur 3 pairs: (i) IR cluster 17 and IR stellar group 18, (ii) W75S IR cluster and IR stellar group 19, and (iii) W $75 \mathrm{~N}$ IR cluster and IR cluster 20. Of 19 single and 3 double embedded IR clusters and stellar groups we derive a binary frequency of $14 \%$ for Cygnus X. This frequency probably reflects star formation and subsequent early dynamical evolution from multiple molecular cores, in a potential dominated by dust and gas.
Despite telescope aperture and seeing limitations with respect to the properties of Cygnus $\mathrm{X}$ clusters, the 2MASS Point Source Catalogue (http:// irsa.ipac.caltech.edu/applications/CatScan/) can provide a preliminary diagnosis on depth effects. We extracted all stars in equal areas for the cluster and offset field, and produced $K_{\mathrm{s}} \times\left(J-K_{\mathrm{s}}\right)$ CMDs. According to the 2MASS extractions the photometric errors are approximately $\epsilon K_{\mathrm{s}}=0.03$ and $\epsilon\left(J-K_{\mathrm{s}}\right)=0.05$ at $K_{\mathrm{s}}=12.45$ (typical 10th brightest star level). Close to the detection level at $K_{\mathrm{s}}=15.0$ the errors increase to $\epsilon K_{\mathrm{s}}=0.18$ and $\epsilon\left(J-K_{\mathrm{s}}\right)=0.26$. The apertures were circular with diameter equal to the major axis (Table 1). We illustrate in Fig. 4 the CMDs of the clusters in DR22 and DR17, together with their respective fields. A significant contrast occurs between the CMDs of cluster and field 

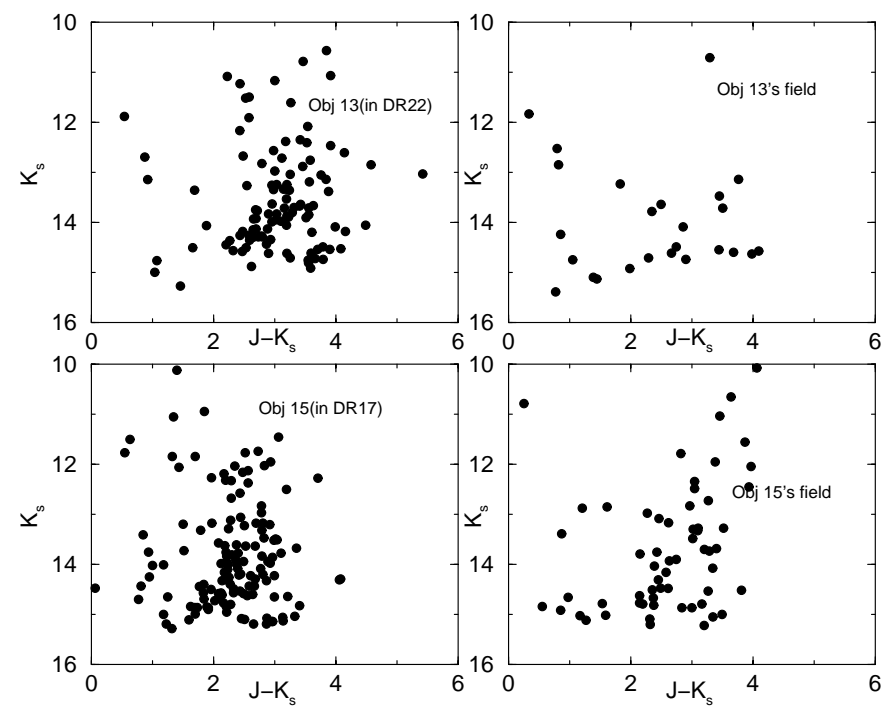

Fig. 4. 2MASS $J \times\left(J-K_{\mathrm{S}}\right)$ colour-magnitude diagrams of the IR clusters in DR22 and DR17. Also shown are those for the equal area offset fields $3^{\prime}$ to the South.

areas. The colour distribution in a cluster CMDs is wide $\left(W\left(J-K_{\mathrm{s}}\right) \approx 1.6 \mathrm{mag}\right)$, as a result of differential reddening and photometric errors. For 7 objects in the Cygnus X area the CMD constrast allowed parameter estimates, for which designations and related nebula are indicated in Table 2 (Cols. 1 and 2, respectively). The remaining objects are affected by nebulosity, crowding and/or the possibility of too faint member stars.

We measured the colour of the upper Main Sequence (Col. 3 of Table 2) and the magnitude of the 10th brightest star in the cluster $\left(J-K_{\mathrm{s}}\right)$ strip (Col. 4 of Table 2$)$. This minimizes field contamination and stellar evolution effects for massive stars. The objects are assumed to be very young embedded clusters, but the results will not change much for relatively evolved clusters up to 6-7 Myr, as long as the 10th brightest star belongs to the MS. Indeed, far infrared and radio observations (Odenwald et al. 1986) suggested ages younger than 0.1 Myr in DR 6 and DR 7, which are predicted to be powered by late $\mathrm{O}$ and early B stars, and DR 22 by an O6 ZAMS star. As $\left(J, K_{\mathrm{s}}\right)$ template we built a 2MASS CMD of NGC 6910, which is itself projected on the eastern side of Cygnus X. It has an age of 6.5 Myr, a reddening value $E(B-V)=1.02$, an absolute distance modulus $(m-M)_{\mathrm{o}}=11.2$ and a distance from the Sun $d_{\odot}=1.74 \mathrm{kpc}$ (Delgado \& Alfaro 2000).

We use the extinction curve by Cardelli et al. (1989) with total-to-selective absorption $R_{V}=3.1$, the $K$ and $K_{\mathrm{s}}$ filter transmissions (Persson et al. 1998) and $A_{K}=0.618$ $E(J-K)$ (Mathis 1990) to derive the relations $A_{K} / A_{V}=$ $0.112, A_{K_{\mathrm{s}}} / A_{V}=0.118$ and $A_{K_{\mathrm{s}}}=0.670 E\left(J-K_{\mathrm{s}}\right)$. The reddening and distance estimates for each cluster were obtained by comparison of the upper Main Sequence colour and 10th brightest star $K_{\mathrm{s}}$ magnitude in the CMDs of the cluster and the template. Columns 5 and 6 of Table 2 list the upper Main Sequence colour difference $\Delta\left(J-K_{\mathrm{S}}\right)$ with respect to the template (in the sense NGC6910Object), and the resulting reddening, respectively. The re-
Table 2. Reddening and distance for Cygnus X embedded objects.

\begin{tabular}{lccccccc}
\hline \hline Cluster & $\begin{array}{c}\text { Rel. } \\
\text { Neb. }\end{array}$ & $\begin{array}{c}\left(J-K_{\mathrm{s}}\right) \\
\mathrm{uMS}\end{array}$ & $\left(K_{\mathrm{s}}\right)$ & $\Delta\left(J-K_{\mathrm{s}}\right)$ & $E\left(J-K_{\mathrm{s}}\right)$ & $(m-M)_{\mathrm{o}}$ & $\begin{array}{c}d_{\odot} \\
(\mathrm{kpc})\end{array}$ \\
\hline NGC 6910 & - & 0.45 & 11.65 & 0.00 & 0.56 & 11.20 & 1.7 \\
Obj.6 & DR9 & 2.50 & 12.95 & 2.05 & 2.61 & 11.13 & 1.7 \\
Obj.7 & DR6 & 2.90 & 12.50 & 2.45 & 3.01 & 10.41 & 1.2 \\
Obj.8 & DR13 & 2.60 & 12.80 & 2.15 & 2.71 & 10.91 & 1.5 \\
Obj.11 & DR7 & 2.55 & 12.10 & 2.10 & 2.66 & 10.25 & 1.1 \\
Obj.13 & DR22 & 3.20 & 12.40 & 2.75 & 3.32 & 10.11 & 1.0 \\
Obj.15 & DR17 & 2.40 & 12.10 & 1.95 & 2.51 & 10.35 & 1.2 \\
Obj.21 & DR16? & 2.55 & 13.10 & 2.10 & 2.66 & 11.25 & 1.8 \\
\hline
\end{tabular}

sulting absolute distance modulus and distance are given in Cols. 7 and 8, respectively. The 10th brightest star in the template cluster NGC 6910 corresponds to an absolute magnitude $M_{K_{\mathrm{s}}}=0.07$. Deeply embedded regions tend to have $R_{V}$ values higher than the standard one (e.g. Comerón \& Torra 1999 and references therein). Nevertheless the use of infrared bands minimizes reddening uncertainties. The upper Main Sequence colour half width $W\left(J-K_{\mathrm{S}}\right) / 2=0.8$ (Fig. 4) converts to absorption error $\epsilon\left(A_{K_{\mathrm{s}}}\right)= \pm 0.54$ which in turn gives a distance error $\approx \pm 0.4 \mathrm{kpc}$ at $d_{\odot} \approx 1.5 \mathrm{kpc}$.

The Cygnus $\mathrm{X}$ clusters in Table 2 are in the range $1.0<d_{\odot}(\mathrm{kpc})<1.8$, with an average distance $d_{\odot} \approx$ $1.36 \pm 0.29 \mathrm{kpc}$. The values suggest a depth of $800 \mathrm{pc}$. The resulting linear ratios along major and minor axes (along the sky at the derived average distance), and depth are $3: 1: 11$. The objects are at a comparable distance or foreground to NGC 6910, and Cyg OB2 also estimated to be located at $d_{\odot} \approx 1.7 \mathrm{kpc}$ (Massey \& Thompson 1991). The derived distance range for Cygnus X suggests a more compact distribution than previous estimates, but still larger than the largest OB associations. At least part of it could be consistent with star formation arising from a local coherent physical event, probably related to Cyg OB2 and NGC 6910. Deep photometry for the present clusters (Table 2) and the additional ones in the Cygnus X direction (Table 1), coupled to high quality spectroscopy for the knowledge of spectral types, may shed further light on the depth issue and history of star formation in the area.

\subsection{Galactic Centre}

Table 1 contains 7 new IR clusters or candidates towards the central areas of the Galaxy. The angular distribution of the previous literature and present objects is shown in Fig. 5. Most of them are outside the $5^{\circ}$ radius region which converts to $\approx 600 \mathrm{pc}$ at the distance of the Galactic centre. Two new objects are located within $600 \mathrm{pc}$, totaling now 60 cluster candidates. The asymmetry of the distribution with respect to the Galactic Nucleus in Fig. 1 is probably an artifact since the region $356<\ell\left(^{\circ}\right)<359$ has not yet been systematically surveyed. Added to the candidates are the well-known central clusters: Arches (Nagata et al. 1993, 1995), Quintuplet (Nagata et al. 1990; Glass et al. 1990) and the Young Nuclear Cluster of 


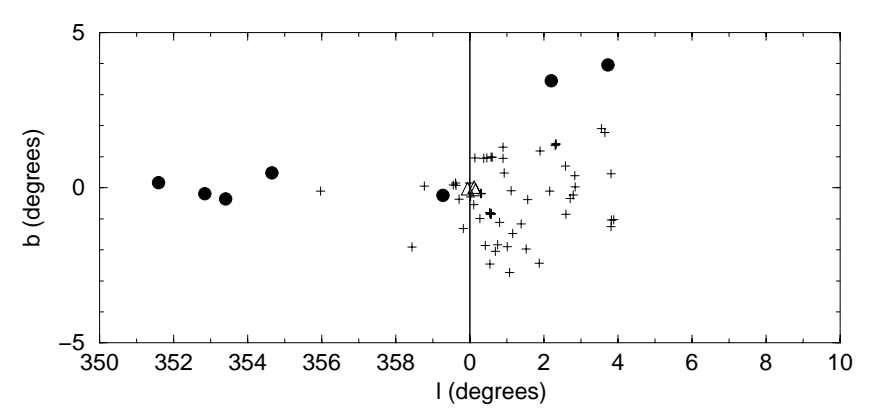

Fig. 5. Angular distribution of the present central clusters and candidates (circles), those from Dutra \& Bica (2000a) (plus signs) and well-known central clusters (triangles).

Table 3. IR Dark nebulae towards the central bulge.

\begin{tabular}{|c|c|c|c|c|c|c|c|}
\hline$\overline{\overline{\text { IRDN }}}$ & $\begin{array}{r}\ell \\
\left({ }^{\circ}\right)\end{array}$ & $\begin{array}{l}{ }^{b} \\
\left({ }^{\circ}\right)\end{array}$ & $\begin{array}{r}(2000) \\
h \text { h:mm:ss }\end{array}$ & $\begin{array}{c}\delta(2000) \\
\vdots^{\prime}:{ }^{\prime \prime}\end{array}$ & $\bar{D}$ & $\overline{\bar{d}}$ & $\overline{\overline{\text { Remarks }}}$ \\
\hline$\overline{1}$ & 0.25 & -0.47 & $17: 48: 02$ & $-28: 58: 00$ & 15 & 11 & related to Sh2-19? \\
\hline 2 & 0.58 & -0.86 & $17: 50: 20$ & $-28: 53: 00$ & 19 & 14 & related to $\mathrm{Sh} 2-21 ?$ \\
\hline 3 & 358.99 & 0.09 & $17: 42: 51$ & $-29: 45: 15$ & 3 & 3 & \\
\hline 4 & 359.34 & 0.29 & $17: 42: 54$ & $-29: 21: 00$ & 5 & 2.5 & \\
\hline 5 & 359.94 & 0.17 & $17: 44: 49$ & $-28: 54: 10$ & 10 & 8 & related to Sh2-17? \\
\hline
\end{tabular}

He I stars (Krabbe et al. 1991). Recently, the latter object was suggested to be the remains of a dissolved young cluster (Gerhard 2001).

During the present study and Paper I we serendipitously came across with prominent dark clouds which are opaque in the $K_{\mathrm{s}}$ band. The clouds are listed in Table 3. Clouds 1, 2 and 5 have larger angular dimensions and are possibly related to intervening HII regions. The dust clouds 3 and 4 are compact with small angular dimensions and are thus candidates to nuclear molecular clouds. Nevertheless, they cannot be ruled out as nearby foreground globules based solely on the images. At any rate, from the dense dust clouds seen in the $K_{\mathrm{s}}$ band projected onto the bulk of the central bulge/disk stellar population, one can infer that a new generation of massive clusters may form in the central disk.

Recently, Portegies Zwart et al. (2001) carried out numerical simulations of the dynamical evolution of massive star clusters within $\approx 200 \mathrm{pc}$ of the Galactic Centre. They concluded that the tidal dissolution time is $\approx 70 \mathrm{Myr}$, but close to the Galactic centre their projected densities drop below the background density within $\approx 20 \mathrm{Myr}$. They estimated that the region within $200 \mathrm{pc}$ could easily harbour as many as 50 massive clusters. Within a circular region with $r<1.6^{\circ}$, or $200 \mathrm{pc}$ assuming a Galactic Center distance $d_{\odot}=8 \mathrm{kpc}$ (Reid 1993), there are 31 clusters or candidates in Fig. 5, thus consistent with the model expectations by Portegies Zwart et al. (2001), taking into account observational and theoretical uncertainties.

In the following we discuss in more detail the objects probably related to an edge-on central disk. The region within $|\ell|=1.6^{\circ}$ and $|b|=0.5^{\circ}$ (Fig. 5) is now relatively well surveyed considering the 3 well-known central clusters, Paper I and the present study samples. It converts to an edge-on cylinder of radius $r \approx 200 \mathrm{pc}$ and distance from the plane $|z|=63 \mathrm{pc}$, where 17 clusters or candidates are projected. From these objects, candidates 1, 17 and 31 (Paper I) are apparently not related to any H II region and might be clusters in the density contrast/tidal survival age ranges $\approx 7-20 \mathrm{Myr}$ or $\approx 7-70 \mathrm{Myr}$. Candidate 58 is possibly related to Sh2-17, and candidates 4, 5 and 6 to Sh2-20. Consequently they are suspected to be intervening objects, and were excluded. The age of the Quintuplet Cluster is $\approx 4$ Myr (Figer et al. 1999), and similar ages can be inferred from the properties of massive stars in the Arches cluster (Lang et al. 2001) and the Young Nuclear cluster (Gerhard 2001; Krabbe et al. 1991). The candidates 26 and 52 are possibly related to the radio H II region Complexes Sgr D and Sgr E (Liszt 1992), respectively. Candidates 53,54 and 55 are possibly related to the radio source G359.54+0.18, candidate 56 to G359.7-0.4, and the present object 42 (Table 1) to G359.3-0.3. Assuming an $\mathrm{HII}$ region duration of $\approx 5 \mathrm{Myr}$ these 10 possible massive clusters imply a formation rate of 2 clusters $\mathrm{Myr}^{-1}$ in the central disk of radius $200 \mathrm{pc}$.

If the dark clouds 3 and 4 in Table 3 are able to form a massive cluster in the coming Myr, they would provide the same rate of cluster formation in the central disk as that derived from the ionising clusters above.

\subsection{Objects throughout the disk}

Several new IR clusters are reported in Table 1 for the anticentre region. In particular 3 new IR clusters are related respectively to the H II regions Sh2-254, Sh2-256 and Sh2-258. These H II regions form a complex together with Sh2-255 and Sh2-257, which are lobes of the bipolar H II region IC 2162. The most prominent IR cluster in the complex Sh2-254/258 was reported by Hodapp (1994) and it is located between the two lobes. The complex now contains 4 clusters and a designation IC 2162 IR cluster or Sh2-255/257 IR cluster is more suitable.

In Table 1 two additional pairs occur: (i) the IR cluster 33 and the stellar group 34, (ii) the IR cluster 24 and Sh2233SE IR cluster (Hodapp 1994). Two IR clusters are related to optical reflection nebulae: vdBH-RN26 and vdBHRN43 (van den Bergh \& Herbst 1975). The former appears to be related to the $\eta$ Carinae Complex, and the second to the RCW 38 Complex. They are located in the outskirts of the complexes, and if physically related to them, they would imply formation of less massive star clusters, still embedded in their dust, which have not formed a massive ionising star.

\section{Concluding remarks}

A new sample of 42 IR star clusters, stellar groups and candidates was found on the 2MASS Atlas, together with 1 bright nebula and 5 dark nebulae. Most of the objects are in the Cygnus X area, where a census of embedded clusters and stellar groups appears to be significant now, with 25 entries. A binary frequency among physical stellar groups of $14 \%$ was obtained in Cygnus X. Distances 
were estimated for 7 Cygnus X clusters, providing a depth of $\approx 800$ pc. Such range suggests line-of-sight projections, but it is not excluded that part of that is coherently connected to the Cyg OB2 formation event and subsequent interaction with the surrounding medium.

We found 7 new IR clusters and candidates towards the central parts of the Galaxy. The census of IR clusters and candidates towards these central parts is $\approx 60$ within $600 \mathrm{pc}$, and 31 within $200 \mathrm{pc}$. These numbers are within expectations for the number of central massive star clusters in terms of formation rate and tidal survival in the central parts of the Galaxy (Portegies Zwart et al. 2001). Opaque dark clouds in the 2MASS $K_{\mathrm{s}}$ images of the central parts of the Galaxy led to molecular cloud candidates, perhaps able to form the next generation of massive nuclear star clusters.

Efforts like the present one to identify and determine accurate positions for new embedded star clusters are crucial for observational purposes, since radio HII regions can be so much reddened that in many cases they remain undetectable even in the $K$ band. Positive detections by means of 2MASS can spare observing time in large telescopes, and can shed more light on the population of young embedded clusters in molecular complexes, and clusters in general which are embedded in the Galactic plane.

Acknowledgements. This publication makes use of data products from the Two Micron All Sky Survey, which is a joint project of the University of Massachusetts and the Infrared Processing and Analysis Center/California Institute of Technology, funded by the National Aeronautics and Space Administration and the National Science Foundation. We employed catalogues from CDS/Simbad (Strasbourg) and Digitized Sky Survey images from the Space Telescope Science Institute (U.S. Government grant NAG W-2166) obtained using the extraction tool from CADC (Canada). We acknowledge support from the Brazilian institution CNPq.

\section{References}

Alter, G., Balázs, B., \& Ruprecht, J. 1970, Catalogue of Star Clusters and Associations, 2nd edition (Budapest: Akademia Kiadó)

Beichman, C., Neugebauer, G., Habing, H. J., Clegg, P. E., \& Chester, T. J. 1988, in IRAS Catalog and Atlas, vol. 1, Explanatory Supplement (Washington, DC: GPO)

Bica, E., \& Dutra, C. M. 2000, AJ, 119, 1214

Bica, E., \& Schmitt, H. R. 1995, ApJS, 101, 41

Bica, E., Schmitt, H. R., Dutra, C. M., \& Luz Oliveira, H. 1999, AJ, 117, 238

Blitz, L., Fich, M., \& Stark, A. A. 1982, ApJS, 49, 183

Cardelli, J. A., Clayton, G. C., \& Mathis, J. S. ApJ, 345, 245

Carpenter, J. M. 2000, AJ, 120, 3139

Caswell, J. L., \& Haynes, R. F. 1987, A\&A, 171, 261

Chen, H., Tafalla, M., Greene, T. P., Myers, P. C., \& Wilner, D. J. 1997, ApJ, 475, 163

Comerón, F., \& Torra, J. 1999, A\&A, 349, 605

Comerón, F., \& Torra, J. 2001, A\&A, 375, 539

Delgado, A. J., \& Alfaro, E. J. 2000, AJ, 119, 1848

Downes, D., \& Rinehart, R. 1966, ApJ, 144, 937

Dutra, C. M., \& Bica, E. 2000a, A\&A, 359, L9
Dutra, C. M., \& Bica, E. 2000b, A\&A, 359, 349

Feinstein, A. 1982, AJ, 87, 1012

Figer, D. F., McLean, I. S., \& Morris, M. 1999, ApJ, 514, 202

Gerhard, O. 2001, ApJ, 546, L39

Glass, I. S., Moneti, A., \& Moorwood, A. F. M. 1990, MNRAS, 242,55

Hillenbrand, L. A., Meyer, M. R., Strom, S. E., \& Skrutskie, M. F. 1995, AJ, 109, 280

Hodapp, K. W., \& Rayner, J. 1991, AJ, 102, 1108

Hodapp, K. W. 1994, ApJS, 94, 615

Knödlseder, J. 2000, A\&A, 360, 539

Krabbe, A., Genzel, R., Drapatz, S., \& Rotaciuc, V. 1991, ApJ, 382, L19

Kuchar, T. A., \& Clark, F. O. 1997, ApJ, 488, 224

Kurtz, S., Churchwell, E., \& Wood, D. O. S. 1994, ApJS, 91, 659

Lada, C. J., Alves, J., \& Lada, E. A. 1996, AJ, 111, 1964

Lang, C. C., Goss, W. M., \& Rodriguez, L. F. 2001, ApJ, 551, L143

Lauberts, A. 1982, The ESO/Uppsala Survey of the ESO (B) Atlas, (Garching: ESO)

Liszt, H. S. 1992, ApJS, 82, 495

Lockman, F. J. 1989, ApJS, 71, 469

Lynds, B. T. 1962, ApJS, 7, 1

Lyngå, G. 1987, Catalogue of Open Clusters (Strasbourg: CDS)

Massey, P., \& Thompson, A. B. 1991, AJ, 101, 1408

Mathis, J. S. 1990, ARA\&A, 28, 37

Moore, T. J. T., Mountain, C. M., Yamashita, T., \& Selby, M. J. 1988, MNRAS, 234, 95

Moorwood, A., Cuby, J. G., Biereichel, P., et al. 1998, Msngr, 94,7

Nagata, T., Woodward, C. E., Shure, M., Pipher, J. L., \& Okuda, H. 1990, ApJ, 351, 83

Nagata, T., Hyland, A. R., Straw, S. M., Sato, S., \& Kawara, K. 1993, ApJ, 406, 501

Nagata, T., Woodward, C. E., Shure, M., \& Kobayashi, N. 1995, AJ, 109, 1676

Norris, R. P., Booth, R. S., \& Davis, R. J. 1980, MNRAS, 190, 163

Odenwald, S., Shivanandan, K., Campbell, M., et al. 1986, ApJ, 306, 122

Odenwald, S. F., \& Schwartz, P. R. 1993, ApJ, 405, 706

Persson, S. E., Murphy, D. C., Krzeminski, W., Roth, M., \& Rieke, M. J. 1998, AJ, 116, 2475

Piepenbrink, A., \& Wendker, H. J. 1988, A\&A, 191, 313

Portegies Zwart, S. F., Makino, J., McMillan, S. L. W., \& Hut, P. 2001, ApJ, 546, L101

Reid, M. 1993, ARA\&A, 31, 345

Rodgers, A. W., Campbell, C. T., \& Whiteoak, J. B. 1960, MNRAS, 121, 103

Saurer, W., Seeberger, R., Weinberger, R., \& Ziener, R. 1994, AJ, 107, 2101

Sharpless, S. 1959, ApJ, 4, 257

Skrutskie, M., et al. 1997, in The Impact of Large Scale NearIR Sky Surveys, ed. Garzon, et al. (Dordrecht: Kluwer), 25

Setteducati, A. F., \& Weaver, M. F. 1962, Newly Found Stellar Clusters (Berkeley: University of California)

van den Bergh, S., \& Herbst, W. 1975, AJ, 80, 208

Vázquez, R. A., Baume, G., Feinstein, A., \& Prado, P. 1996, A\&AS, 116, 75

Wendker, H. J. 1970, A\&A, 4, 378

Wendker, H. J. 1984, A\&AS, 58, 291

Wendker, H. J., Higgs, L. A., \& Landecker, T. I. 1991, A\&A, 241,551 